This item was submitted to Loughborough's Research Repository by the author.

Items in Figshare are protected by copyright, with all rights reserved, unless otherwise indicated.

\title{
Characterisation of wake bi-stability for a square-back geometry with rotating wheels
}

PLEASE CITE THE PUBLISHED VERSION

https://doi.org/10.1007/978-3-319-67822-1_6

PUBLISHER

Springer

VERSION

AM (Accepted Manuscript)

\section{PUBLISHER STATEMENT}

This work is made available according to the conditions of the Creative Commons Attribution-NonCommercialNoDerivatives 4.0 International (CC BY-NC-ND 4.0) licence. Full details of this licence are available at: https://creativecommons.org/licenses/by-nc-nd/4.0/

\section{LICENCE}

CC BY-NC-ND 4.0

\section{REPOSITORY RECORD}

Pavia, Giancarlo, and Martin A. Passmore. 2018. "Characterisation of Wake Bi-stability for a Square-back Geometry with Rotating Wheels”. figshare. https://hdl.handle.net/2134/35518. 


\title{
6 Characterisation of wake bi-stability for a square-back geometry with rotating wheels
}

Giancarlo Pavia, Martin Passmore

\begin{abstract}
In this paper the effects produced by the wheels on the bi-stable reflectional symmetry breaking (RSB) mode seen for the wake of a square-back geometry (Grandemange et al. [11]) are investigated considering a modified version of the Windsor body already studied in Perry et al. [18]. The contribution of the wheels and their rotation to the changes in the base pressure distribution and the wake topology is characterised by means of pressure tappings and 2D-3C particle image velocimetry. Balance measurements are used to further characterise the changes in the strength of the RSB mode. For the pure square-back configuration, the results show a general increase of the base drag as a consequence of the strengthening of the suction over the lower portion of base, due to the formation of a pair of counter rotating vortices acting close to the bottom trailing edge. At the same time, the RSB mode is weakened, leading to a reduction in the fluctuations recorded for the lateral component of the aerodynamic force. The sensitivity of the RSB mode to small changes in the shape of the model's trailing edges is characterised by looking at the effects produced by short tapers, with a slant angle of $12^{\circ}$ and a chord equal to $4 \%$ of the model length, applied to either the horizontal or the vertical trailing edges. The results show that the RSB mode disappears when the effect of the wheels is paired to the upwash generated by the slanted surface (when applied to the bottom trailing edge), although it is still clearly visible when the tapers are applied to the side edges of the base, in contrast with the results reported by Pavia et al. [16] for the same geometry without wheels.
\end{abstract}




\section{Introduction}

Square-back vehicles are popular in the automotive industry thanks to their large cabin space and easy access. The large base area, however, yields a higher level of aerodynamic drag compared to other more streamlined rear-end shapes such as those used in fast-back or notch-back cars.

The steady state topology of the wake generated by such geometries has been fully characterised, from both a numerical (Krajnovic and Davidson [14]) and experimental (Perry et al., [17]) point of view. It consists of a toroidal structure which is source of significant pressure losses and whose interaction with the surrounding shear layers results in the formation of a pair of counter rotating vortices downstream of the near wake region. Obtaining accurate results from the numerical simulations of this vortical structure, however, is still an open challenge (Makihara et al. [15]), due to the complex dynamics that accompany the development of the wake itself.

Grandemange et al. [11], testing a 1/4 scale Ahmed body (see Ahmed et al. [1]), pointed out that the wake of a square-back geometry is characterised by the coexistence of two different types of coherent motions. The first is a set of global fluctuating modes, consisting of a lateral and vertical flapping as well as a longitudinal pumping (all with a characteristic time of $\mathrm{T}_{\mathrm{s}} \sim 5 \mathrm{H} / \mathrm{V}_{\infty}$ as confirmed by Volpe et al. [23]). The second type of motion is a bi-stable reflectional symmetry breaking (RSB) mode, whose characteristic time is about three orders of magnitude longer than the first one $\left(\mathrm{T}_{1} \sim 10^{3} \mathrm{H} / V_{\infty}\right.$, with $V_{\infty}$ the free-stream velocity). The succession of RSB states has been shown to behave like a weak chaotic process with two attractors (Varon et al. [22]). It leads to large variations in the values of side force that have been shown to be responsible for part of the drag experienced by the model (Grandemange et al. [12]).

A similar trend has been also noticed by Grandemange et al. [9] testing an Ahmed body scaled to a full size small car (resulting in a model height based Reynolds number of $\operatorname{Re}_{\mathrm{H}}=2.5 \cdot 10^{6}$ ) and equipped with four rotating wheels. When the model was tested for values of the yaw angle $\Psi$ between $-0.5^{\circ}$ and $+0.5^{\circ}$, large fluctuations were noticed in the signal related to lateral component of the aerodynamic force recorded at $10 \mathrm{~Hz}$ for $40 \mathrm{~s}$, whilst the drag remained constant in the same range.

The same dependency between $C_{D}$ and $\Psi$ was found by Bonnavion et al. [4] while assessing the presence of bi-stability on the wake generated by a commercially available light van. They reported the existence of a bi-stable regime between two different positions of the reverse flow impinging on the base when the model was at $4^{\circ}$ yaw. In this case, however, the RSB mode was found to have an affect mainly on the vertical component of the aerodynamic force, as the model high was greater than the width, in agreement with the conclusions drown by Grandemange et al. [10] studying the effects of different values of the base aspect ratio on the RSB mode. 
A deeper understanding of the bi-stable behaviour has been achieved while also developing suitable strategies to control this type of instability. Grandemange et al. [12] suggested that a base drag reduction between $4 \%$ and $9 \%$ can be achieved for an Ahmed body when the lateral symmetry of the wake is restored by means of a vertical control cylinder positioned in the core of the wake's reverse flow region. An even bigger improvement was reported by Evrard et al. [7] applying a cavity to the base of a similar model; the RSB mode was weakened as the depth of the cavity (d) was increased, until saturation was reached for $d>0.24 \mathrm{H}$. A smaller improvement (around $2 \%$ over the uncontrolled case) has been seen from the feedback control system developed by Brackston et al. [5].

Grandemange et al. [12] also found that the RSB mode disappears when the horizontal symmetry of the time averaged wake is broken by perturbing one of the two horizontal shear layers with a control cylinder placed along the same direction, yielding noticeable variations in the lift over the uncontrolled case. A similar trend was noticed by Barros [2] studying the sensitivity of the wake past a square-back Ahmed body to steady perturbations applied underneath the model. He noticed that a perfect bi-modal behaviour along the transverse direction can be seen only when the steady state wake topology shows a good level of symmetry along vertical direction. The RSB mode, in fact, tends to weaken until disappearing as the horizontal symmetry is progressively lost. This agrees with the behaviour found by Perry et al. [18] looking at the effects produced by high aspect ratio tapers applied to the top and bottom trailing edges of a Windsor body, with the aim of replicating the shape optimisation process that is common for many modern production vehicles. It was seen that the tendency of the wake to develop a bi-stable behaviour tends to decrease as the distance between the top and bottom shear layers is reduced by the upwash or downwash induced by the small slants. However, a reduction of the drag generated by the rear facing surfaces $C_{D_{R e a r}}$ was obtained only when the weakening of the RSB mode was accompanied with the re-symmetrisation of the time averaged wake topology along the vertical direction. An even bigger improvement of $C_{D_{R e a r}}$ was achieved by Pavia et al. [16] forcing the lateral symmetry of the wake by applying similar tapers to the vertical trailing edges of the same model, leaving the horizontal edges squared.

The RSB mode and its effects on the base drag as well as the lateral force experienced by the model are further investigated in the present work by considering a geometry that, although being still fairly simple, is more representative of a real vehicle than those considered for example in Grandemange et al. [11] and Perry et al. [18], thanks to the presence of four rotating wheels. 


\section{Experimental Methodology}

\subsection{The Windsor Body}

A Windsor body similar to that used in the work of Perry et al. [18] has been considered. The model is a simplified geometry, approximately equivalent to a $1 / 4$ scale passenger car (Length $L=1044 \mathrm{~mm}$, width $\mathrm{W}=389 \mathrm{~mm}$, height $\mathrm{H}=289 \mathrm{~mm}$ ), resulting in a tunnel blockage of $4.4 \%$.

In the case considered in the present work, the model has been equipped with four rotating aluminium wheels, machined from solid. Each wheel has an external diameter $\mathrm{D}_{\mathrm{w}}$ of $150 \mathrm{~mm}$ and a maximum width of $\mathrm{W}_{\mathrm{w}}$ of $55 \mathrm{~mm}$. The front axle is placed $280 \mathrm{~mm}$ upstream of the centre of the model while the rear axle is located $357 \mathrm{~mm}$ downstream of the same point, resulting in a wheelbase I of $637 \mathrm{~mm}$, with the front and the rear overhangs measuring respectively $l_{f}=242 \mathrm{~mm}$ and $l_{r}=165$ $\mathrm{mm}$. The torque needed to spin the wheels was provided by four electrical brushless motors, with an external diameter $\mathrm{D}_{\mathrm{m}}$ of $60 \mathrm{~mm}$ and a maximum width of $\mathrm{W}_{\mathrm{m}}$ of 48 $\mathrm{mm}$, mounted inside each wheel. The wheels were housed inside four 3D printed wheel arches, with a diameter $\mathrm{D}_{w a}$ of $170 \mathrm{~mm}$ and a depth of $96 \mathrm{~mm}$ at the front and $76 \mathrm{~mm}$ at the rear. When the wheels were not in use, the wheel arches were replaced with four $3 \mathrm{D}$ printed covers mounted flush with the side and underbody surfaces.

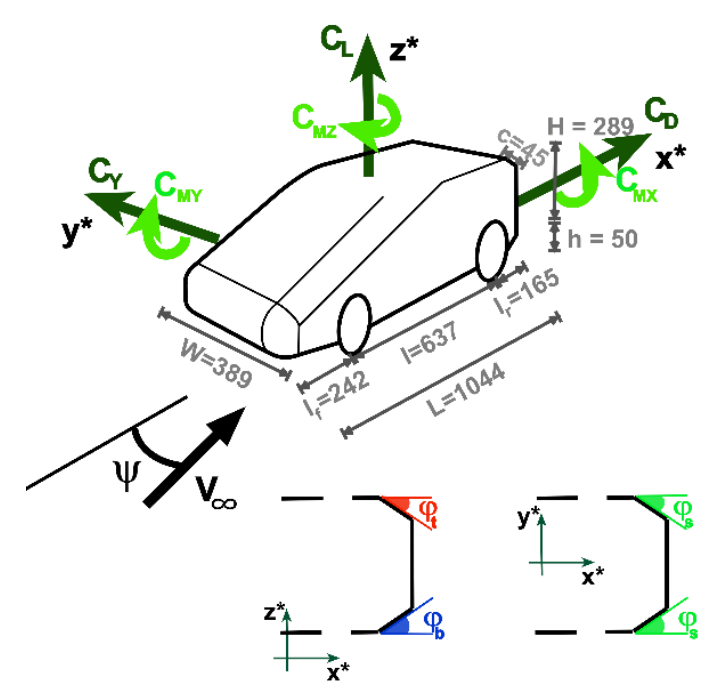

(a)

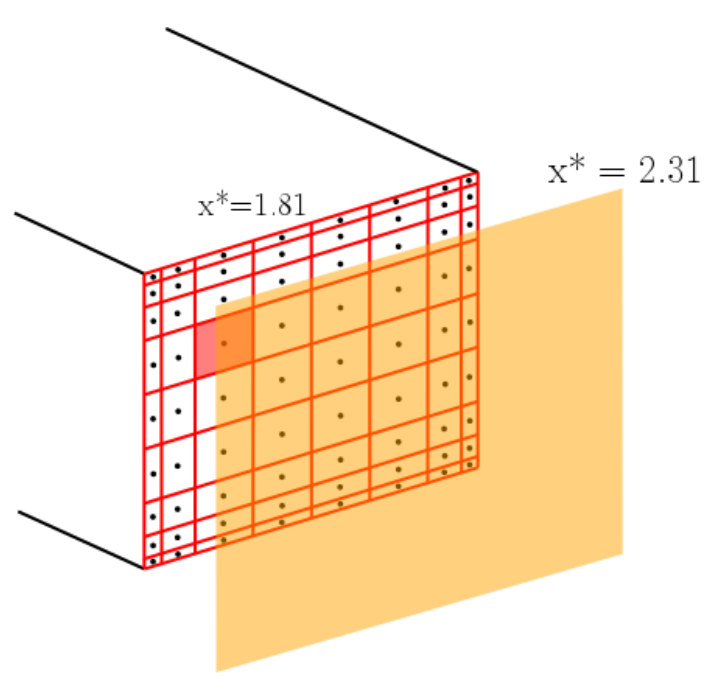

(b)

Fig. 1: a schematic representation of the Windsor body; $\mathbf{b}$ representation of the model base with the pressure tappings and the 2D-3C PIV plane considered in the present work. The red square on the model base sketched in $\mathbf{b}$ denotes the area associated with the $\mathrm{n}^{\text {th }}$ tap used for the estimation of the area weighted drag. All the dimensions reported in a are expressed in $\mathrm{mm}$. For the reference system see SAE [20]. 
The model is also equipped with a removable rear section that allows testing of multiple rear taper configurations. Each taper has a chord c of $45 \mathrm{~mm}$. Besides the pure square-back configuration, four cases have been studied, based on the results of Pavia et al. [16]: in the first two cases a slant with an angle of $12^{\circ}$ was applied to either the upper or lower horizontal trailing edge, leaving the opposite edge squared, whilst two more tests were performed applying the same slant to both the horizontal or the vertical trailing edges. For the sake of clarity, $\phi_{t}$ denotes the angle formed by the top taper with the flat section of model roof, $\phi_{b}$ represents the angle between the bottom chamfer and the flat model underbody, whereas $\phi_{s}$ indicates the angle formed between each vertical taper and flat lateral surface of the model (see Fig. 1a).

The model was mounted via four pins (M8 threaded bar) to the six component balance located beneath the working section floor. The pins were placed between the two axles, $27.5 \mathrm{~mm}$ inboard of the model sides. The wheels were mounted separately from the model, bolted to the balance turntable on purpose-designed steel brackets.

For all the tests the ground clearance was set at $50 \mathrm{~mm}$ (17.3\% of the model height), with a tolerance of $0.2^{\circ}$ for the pitch angle. The contact between the wheels and the ground was represented with a $3 \mathrm{~mm}$ deep machined scallop underneath each wheel.

The SAE coordinate system (SAE [20]) is used throughout; the $X$ axis is aligned with the flow in the downstream direction, the $Z$ axis is vertical, positive upwards, and the $Y$ axis follows a right handed coordinate system. The origin is on the ground plane at mid wheelbase, mid track (Fig. 1a). All the quantities presented throughout the paper have been normalised using the model height $\mathrm{H}$ as the reference length and the value of the free stream velocity $V_{\infty}$ and are denoted with the superscript *.

\subsection{The Wind Tunnel}

All experiments were carried out in the Loughborough University Large Wind Tunnel. This test facility, described in Johl [13], has a working section measuring $1.92 \times 1.32$ $\times 3.6 \mathrm{~m}\left(\mathrm{~W}_{\mathrm{T}} \times \mathrm{H}_{\mathrm{T}} \times \mathrm{LT}_{\mathrm{T}}\right)$ and is equipped with a fixed floor without any upstream boundary layer treatment. In empty conditions, the free stream turbulence level inside the test section is approximately $0.2 \%$, with a flow uniformity of $\pm 0.4 \%$ of the mean flow value. In this state, the boundary layer thickness at the model origin was measured to be equal to $\delta_{99}=64 \mathrm{~mm}$. All tests were performed with a free stream velocity of $40 \mathrm{~m} / \mathrm{s}$, corresponding to a Reynolds number $\mathrm{Re}_{H}$ of $7.7 \times 10^{5}$ based on the model height. When spinning, the rotational speed of the wheels was set manually to match the tunnel free stream velocity, with an accuracy of \pm 50 RPM. 


\subsection{Balance Measurements}

The aerodynamic loads acting on the main body were recorded by means of an Aerotech ${ }^{\circledR}$ six-component virtual centre balance, located under the working section of the tunnel. The balance features analogue to digital conversion at the load cell to minimise signal degradation, and an automated yaw mechanism with a positional accuracy of $0.1^{\circ}$. Further information can be found in Johl [13]. The aerodynamic loads were sampled at $100 \mathrm{~Hz}$ for $630 \mathrm{~s}$. Prior to logging the data, a $30 \mathrm{~s}$ settling time was used for all measurements. The recorded values of the forces have been normalised using Eq. 1:

$$
C_{F i}=\frac{\text { Force }}{0.5 \rho S V_{\infty}^{2}}
$$

where $\rho$ is the air density and $S$ is the projected model frontal area $\left(S=0.1124 \mathrm{~m}^{2}\right)$. The coefficients were corrected for blockage effects using Eq. 2:

$$
C_{F i_{C o r r}}=C_{F i}(1-B)^{2}
$$

where $\mathrm{B}$ denotes the blockage value, given by the ratio between the model frontal area $\mathrm{S}$ and the tunnel working section cross sectional area $S_{T}$.

For the sake of simplicity, only the results related to the side force $C_{Y}$ are presented in this work as this is the component of the aerodynamic force most affected by the action of the RSB mode (Grandemange et al. [9] and Grandemange et al. [12]).

\subsection{Pressure Measurements}

The pressure acting on the model base and tapers was recorded by populating the rear facing surfaces with a grid of pressure taps connected via flexible tubes to a pair of Che $\|^{\circledast}$ CANdaq miniature pressure scanners (with a manufacturer quoted accuracy of $\pm 1.47 \mathrm{~Pa}$ ) mounted inside the model. Each scanner is equipped with 64 piezo-resistive pressure sensors, paired to temperature sensors to allow the correction of inaccuracies introduced by temperature drift. Between 72 and 126 taps were used, depending on the configuration. The taps were placed with a finer distribution close to the model edges, to get a more accurate representation of the pressure distribution in the regions with the highest gradients (see Fig. 1b). Data was recorded at $260 \mathrm{~Hz}$ for $630 \mathrm{~s}$. The free-stream dynamic and static pressures were acquired $1870 \mathrm{~mm}$ upstream of the model, at the start of the working section. Signal was corrected for distortion applying the correction proposed by (Sims-Williams and 
Dominy [21]). Once the pressure coefficients were calculated, Eq. 3, the results were corrected for blockage using the MIRA correction (based on continuity), Eq. 4:

$$
\begin{gathered}
C_{p}=\frac{p-p_{\infty}}{0.5 \rho S V_{\infty}^{2}} \\
1-C_{p_{\text {Corr }}}=\left(1-C_{p}\right)(1-B)^{2}
\end{gathered}
$$

where $p_{\infty}$ is the freestream static pressure. The contribution of the model rear facing surfaces to the aerodynamic force experienced by the model was estimated by integrating the measured pressure field:

$$
C_{D_{\text {Rear }}}=-\frac{1}{S} \int_{S} C_{p} \cdot d S \simeq-\frac{1}{S} \sum_{i=1}^{N_{\text {tap }}} C_{p_{i}} S_{i}
$$

where $C_{p_{i}}$ is the time averaged value of the pressure coefficient recorded by the $\mathrm{n}^{\text {th }}$ tap and $S_{i}$ is the projection of the associated area (see Fig. 1b).

\subsection{PIV Measurements}

Stereoscopic particle image velocimetry (PIV) measurements were performed considering a plane orthogonal to the free stream velocity, at $x^{*}=2.31$ from the origin of the reference system (see Fig. 1b). Two LaVision ${ }^{\circledR}$ CMOS cameras with a resolution of $2560 \times 2160$ pixels and a pixel size of $6.5 \times 6.5 \mu \mathrm{m}^{2}$ were employed, together with a Litron ${ }^{\circledR} 200 \mathrm{~mJ}$ double pulsed Nd:YAG laser and PIVTec 45 atomiser, generating $1 \mu \mathrm{m}$ droplets of DEHS. The cameras were mounted on a pair of aluminium rails placed inside the tunnel working section, following the arrangement used by Wood [25]. The separation angle between the cameras was $\approx 50^{\circ}$. Each sensor was equipped with a Nikon ${ }^{\circledR}$ lens, with a focal length of $50 \mathrm{~mm}$, mounted on a tilt system in order to satisfy the Scheimplfug criterion (Prasad [19]). The aperture was set at $\mathrm{f}_{\#}=4$. The resultant field of view $\left(W_{f} \times H_{f}\right)$ was $520 \times 400 \mathrm{~mm}$.

Data acquisition was performed at $15 \mathrm{~Hz}$. During each acquisition, 2000 statistically independent image pairs were captured, matching the total sampling time used by Perry et al. [18] (133.3 s). The data was post-processed using DaVis 8.2.0. Image pre-processing was applied to the recordings in order to mitigate the effects of image distortion, background light intensity and spurious reflections. A calibration correction based on a pinhole model was applied, background subtraction was performed and the outmost regions of the field of view were discarded using a geometric mask. The 
size of the field of view was therefore reduced to $430 \times 340 \mathrm{~mm}$. A multi-pass scheme for cross-correlation was then applied (Willert and Gharib [24]) starting with an interrogation window with a size of $64 \times 64$ pixels and a 50\% overlap between cells and ending with windows of $32 \times 32 \mathrm{~mm}$ pixels and a $75 \%$ overlap. This resulted in a spatial resolution of $2.8 \times 10^{-6} \mathrm{~m}^{2}$. The level of uncertainty associated with the measurements was estimated to be $0.5 \%$ and $1.5 \%$ of the mean and RMS values of the free stream velocity with a $99 \%$ confidence level (Benedict and Gould, [3]).

\section{Results and Discussion}

\subsection{Square-back configuration: effects of the wheels on the RSB mode}

A first series of tests was performed considering the same square-back configuration without wheels studied in Perry et al. [18]. Stereoscopic PIV was performed inside the near wake, $145 \mathrm{~mm}$ downstream of the model base $\left(x^{*}=2.31\right.$ from the origin of the reference system defined in Fig. 1a). The time averaged results, reported in Fig. 2a, show a good level of symmetry along the vertical direction but a slight asymmetry between the two lateral sides of the model, in agreement with that shown by the horizontal 2D PIV presented in Perry et al. [18]. Two reflectional symmetry breaking (RSB) states were then extracted, dubbed $L$ state and $R$ state (Fig. $2 \mathrm{~b}$ and $2 \mathrm{c}$ ), by applying the same conditional average technique used in Perry et al. [18]. Each of these two states is characterised by the presence of two counter rotating vortices aligned with one of the two vertical edges, originating a low pressure region that is responsible of the creation of the horizontal pressure gradient already documented in other works (Evrard et al. [7] and Grandemange [8]). The bi-stable mode consists of a random switch between these two reflectional symmetry breaking states. A good level of lateral symmetry in the time averaged wake topology can be obtained only when the acquisition time is long enough to capture the two states for approximately the same number of realisations.

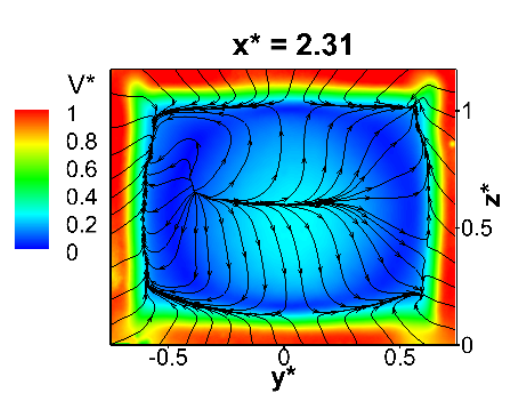

(a)

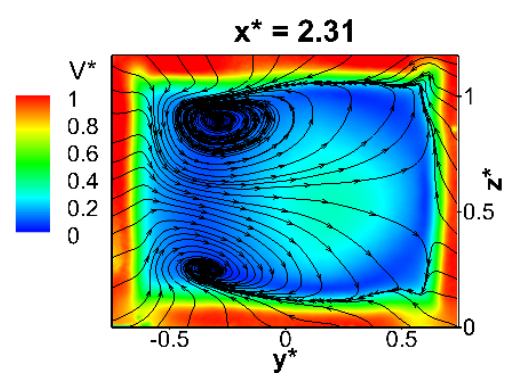

(b)

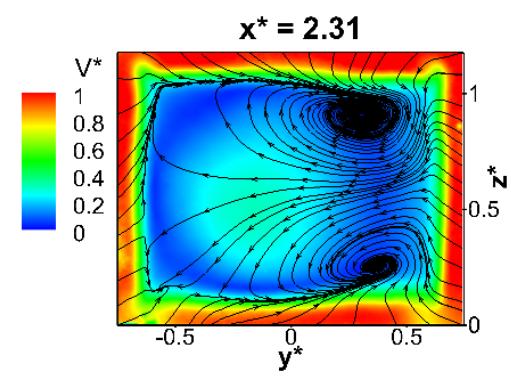

(c)

Fig. 2: Square-back configuration without wheels: a time averaged wake topology for $\mathrm{x}^{*}=2.31 ; \mathbf{b} L$ state and $\mathbf{c} R$ state extracted following the same method used in Perry et al. [18]. 


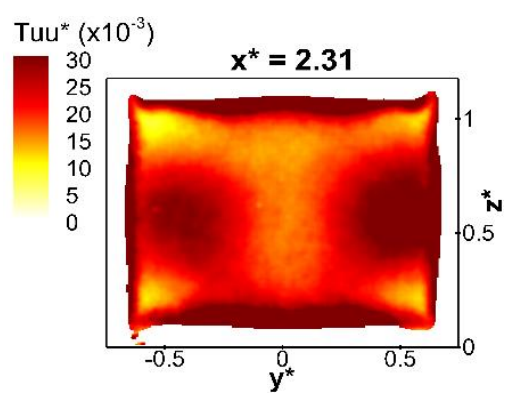

(a)

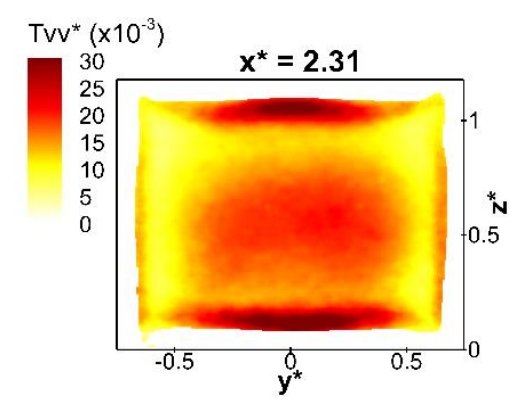

(b)

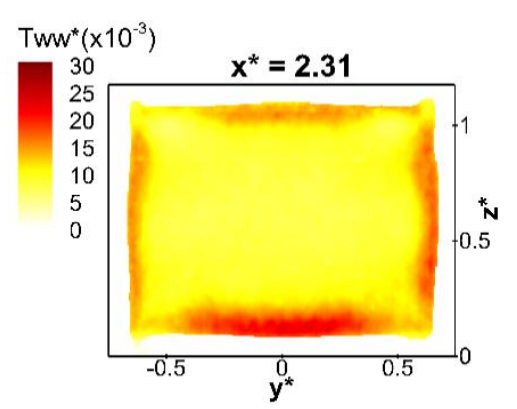

(c)

Fig. 3: Normal components of the Reynolds stress tensor for the square-back model without wheels: $\mathbf{a} \mathrm{T}_{u u^{*}}{ }^{*}=\left\langle\mathrm{u}^{* 2}\right\rangle ; \mathbf{b}_{\mathrm{vv}}{ }^{*}=\left\langle\mathrm{v}^{* \prime 2}\right\rangle ; \mathbf{c} \mathrm{T}_{\mathrm{wv}}{ }^{*}=\left\langle\mathrm{w}^{* \prime 2}\right\rangle$.

Due to the action of this mode, a high level of unsteadiness can be seen close to the core of the wake itself, as shown by the values of the normal components of the Reynolds stresses reported in Fig. 3. In particular, two peaks can be seen in the contour plot obtained for the streamwise component Tuu ${ }^{*}$ (Fig. 3a), corresponding with the centre of each one of the vertical halves of the model base. This matches the distribution of the base pressure fluctuations reported in Pavia et al. [16] for the same model. The level of fluctuations in these regions is comparable with that seen in the shear layers surrounding the wake. A similar intensity is also seen in the lateral component of the Reynolds stresses $T_{v v}{ }^{*}$ recorded in the same area (3b), although this time the peaks are centred with the base and values tend to spread along the horizontal direction rather than being clustered around two lobes. No activity, on the other hand, can be seen in the vertical component $\mathrm{Tww}^{*}$ (Fig. 3c), apart from the regions corresponding to the horizontal and vertical shear layers. All these elements suggest that the RSB mode involves a lateral as well as longitudinal motion of the wake, with the latter being probably related to the pumping motion described by Duell and George [6] and Volpe et al. [23].

The effects produced by the presence of the wheels on the RSB mode as well as the base drag were studied by applying the four wheels described in $\$ 2.1$ to the squareback configuration.

The addition of just the front axle yields a slight decrease in the values of pressure recorded around the lower portion of the base over the square-back configuration tested in Pavia et al. [16] (Fig. 4a). The increase in the suction in this area is counteracted by a better pressure recovery over the upper half of the base, yielding only a marginal variation in the base drag $\left(\Delta C_{D_{\text {Rear }}} \simeq+1\right.$ count). No differences can be noticed between the stationary and the rotating case. The presence of a strong bi-stable motion is still recognisable, as seen from the distribution of the pressure fluctuations over the model base that is in good agreement with the two-lobe distribution shown for example by Volpe et al. [23] for a bi-stable case (Fig. 5a). 


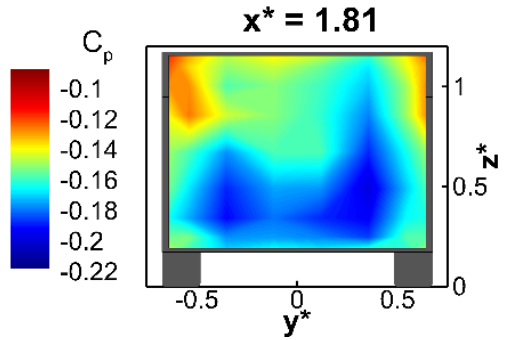

(a)

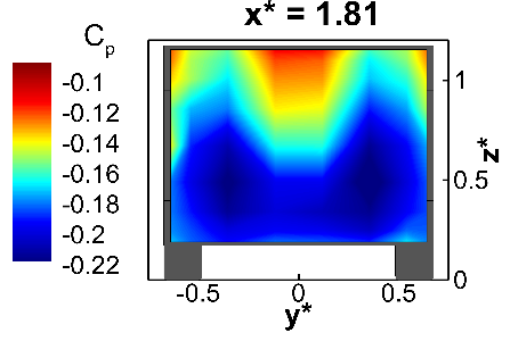

(b)

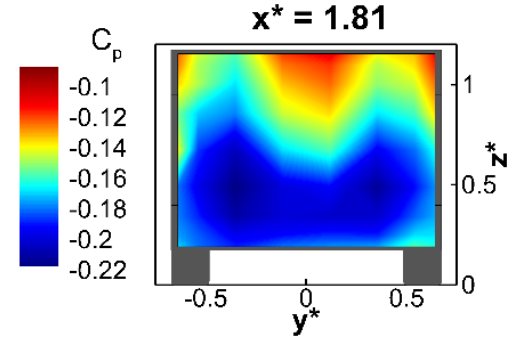

(c)

Fig. 4: Time averaged base pressure distribution for the square-back configuration: a front axle only (with stationary wheels); $\mathbf{b}$ four stationary wheels; $\mathbf{c}$ four rotating wheels.

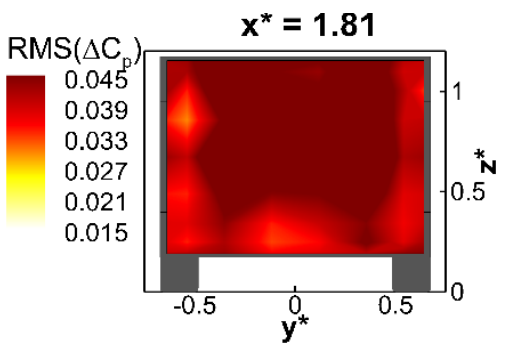

(a)

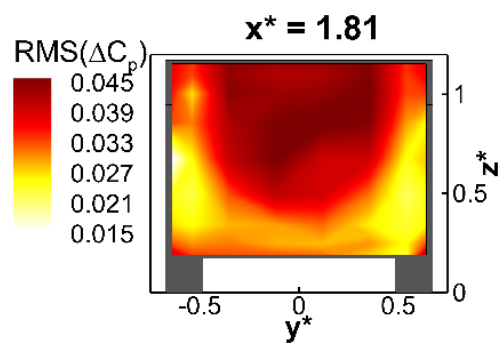

(b)

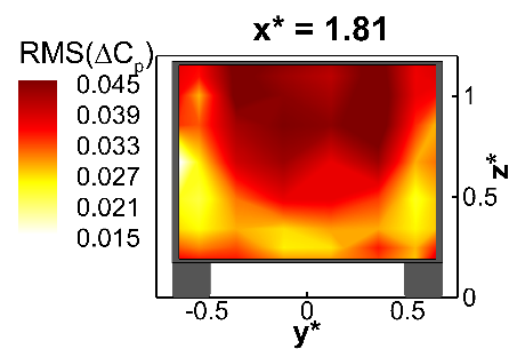

(c)

Fig. 5: Base pressure fluctuations for the square-back configuration: a front axle only (with stationary wheels); $\mathbf{b}$ four stationary wheels; $\mathbf{c}$ four rotating wheels.

A noticeable change in the distribution of the pressure fluctuations can be seen when the rear wheels are added to the model (Fig. 5b and 5c). In this case, the region with the highest level of fluctuations is reduced in size and moved towards the upper edge of the base, in a similar fashion to that reported by Perry et al. [18] when the upwash in the near wake was increased by adding a small taper to the bottom trailing edge of the model. On this occasion, however, the two-lobe structure becomes harder to see inside the region with the highest values of $\operatorname{RMS}\left(\Delta C_{p}\right)$, suggesting an even further weakening of the RSB mode. No significant differences can be seen between the stationary (Fig. 5b) and the rotating (Fig. 5c) case. Similar conclusions hold when comparing the topologies of the time averaged base pressure distributions for the same configurations (Fig. 4b and Fig. 4c). In both cases a further growth of the suction previously noticed in the bottom half of the base can be seen. This results in an increase of $C_{D_{\text {Rear }}}$ between 13 counts and 7 counts over the square-back configuration without wheels, with the rotating configuration showing a better pressure recovery than the stationary case. 


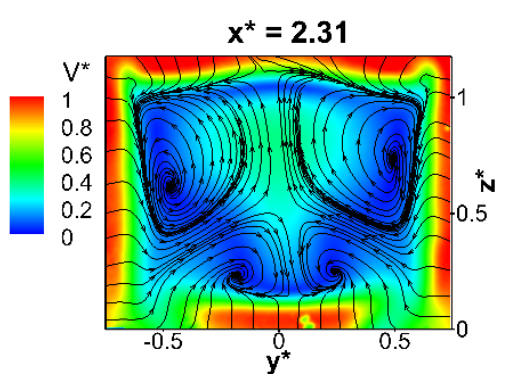

(a)

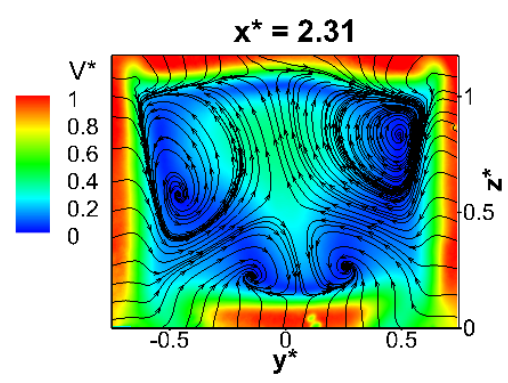

(b)

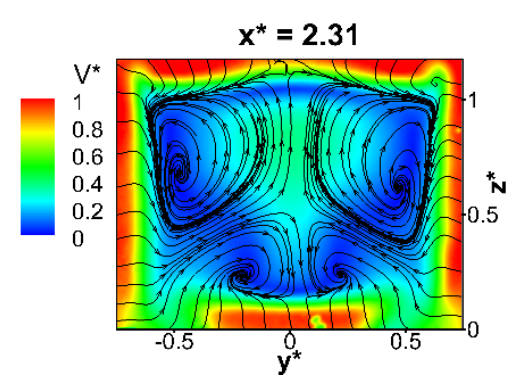

(c)

Fig. 6: Square-back configuration with stationary wheels: a time averaged wake topology for $\mathrm{x}^{*}=2.31 ; \mathbf{b} L$ state and $\mathbf{c} R$ state extracted by applying the same method used in Perry et al. [18].

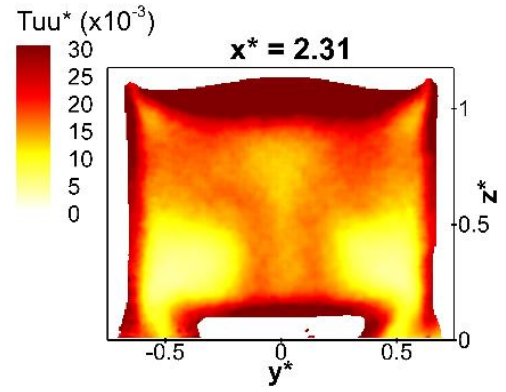

(a)

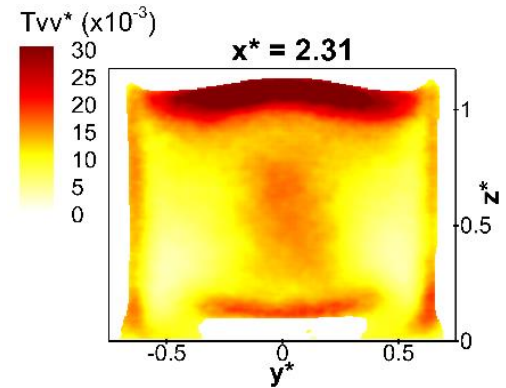

(b)

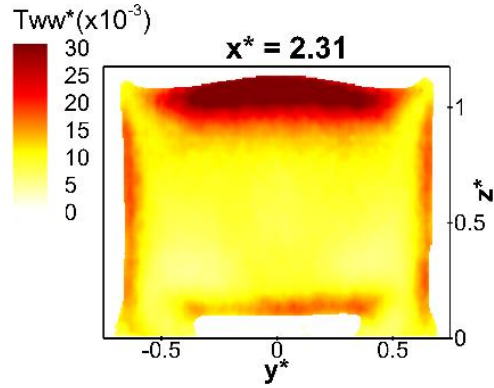

(c)

Fig. 7: Normal components of the Reynolds stress tensor for the square-back model with

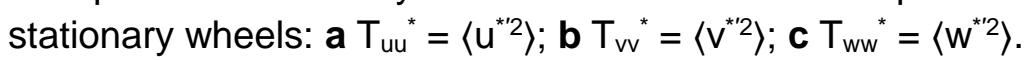

The origin of this low pressure region can be inferred by looking at the time averaged velocity field presented in Fig. 6a. The PIV data shows the presence of a pair of counter rotating vortices acting in the gap between the two rear wheels. These vortices are quite stable, as evidenced by the almost null values of the three normal components of the Reynolds stresses in this region (Fig. 7). This is in contrast to the higher level of instability seen in the pair of recirculating structures acting on the upper portion of the base, although the values obtained for $T_{u u}{ }^{*}$ and $T_{v v}{ }^{*}$ are somehow lower than in the previous case (Fig. 3). All these elements suggest the existence of a stabilising effect on the wake produced by the vortical structures created by the wheels. The action of these vortices appears to counteract the tendency of the upper recirculating structures to align their centres alternately with the two vertical trailing edges, resulting in the RSB states previously described. As a consequence, the lateral "displacement" of the wake is limited and the only difference between the two states, when they are isolated using the method proposed in Perry et al. [18], is in the direction of the reverse flow impinging on the upper portion of the base (Fig. 6b and 6c). 


\subsection{Effects produced by horizontal trailing edge tapers on the RSB mode}

The sensitivity of the wake to small changes in the shape of the model trailing edges was studied by applying small tapered surfaces, with a slant angle of $12^{\circ}$, to the edge of the base. Three configurations are reported: top slant only, bottom only and both top and bottom tapers. All tests are with wheels in place, as already described in §2. For the sake of simplicity only the results related to the stationary case are presented, since having the wheels rotating did not produce noticeable changes in either the unsteady behaviour of the wake or its time averaged topology, as described in 33.1 .

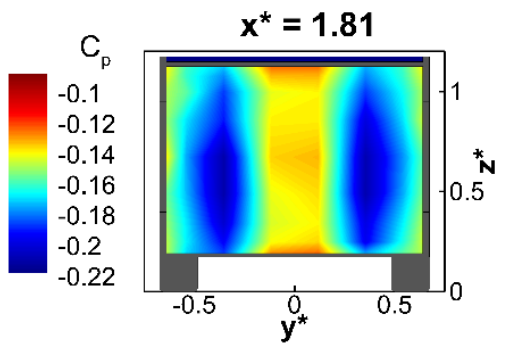

(a)

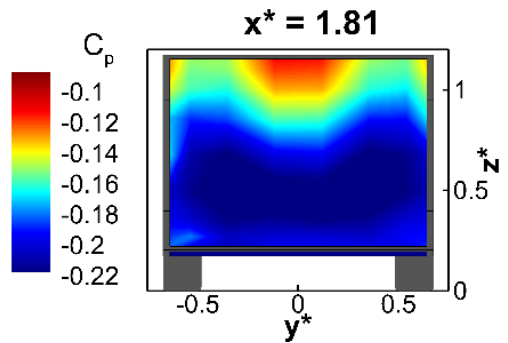

(b)

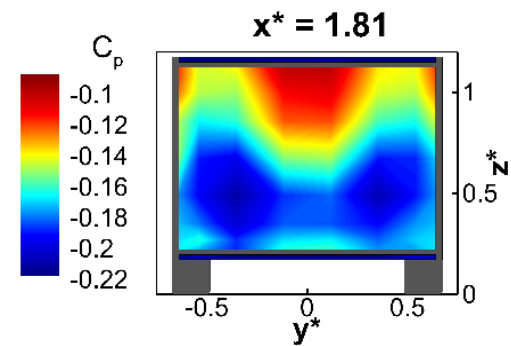

(c)

Fig. 8: Time averaged base pressure distribution for different rear-end configurations with stationary wheels: $\mathbf{a} \phi_{\mathrm{t}}=12^{\circ}, \phi_{\mathrm{b}}=0^{\circ} ; \mathbf{b} \phi_{\mathrm{t}}=0^{\circ}, \phi_{\mathrm{b}}=12^{\circ} ; \mathbf{c} \phi_{\mathrm{t}}=12^{\circ}, \phi_{\mathrm{b}}=12^{\circ}$.

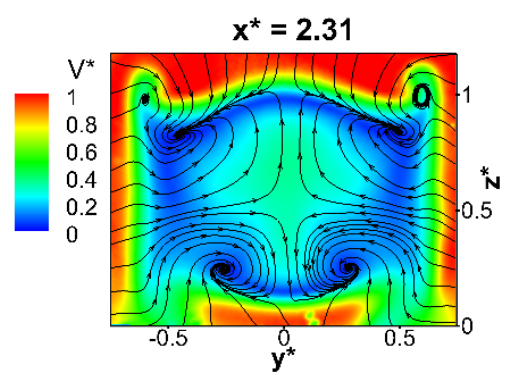

(a)

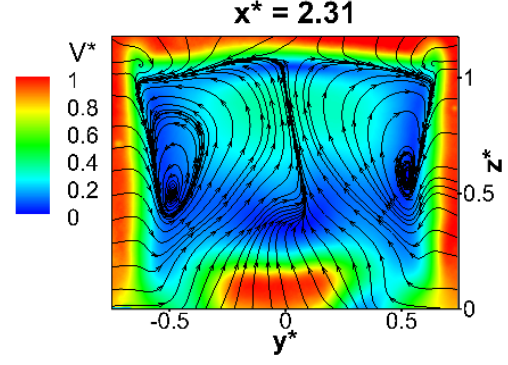

(b)

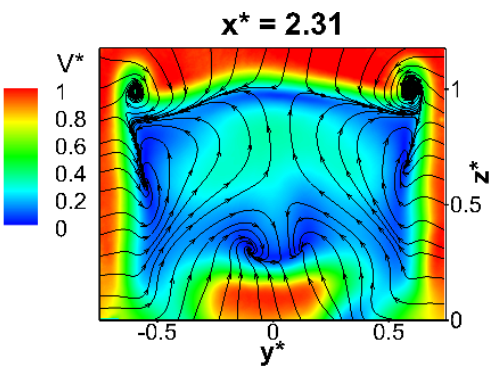

(c)

Fig. 9: Time averaged velocity field at $x^{*}=2.31$ for different rear-end configurations with stationary wheels: a $\phi_{\mathrm{t}}=12^{\circ}, \phi_{\mathrm{b}}=0^{\circ} ; \mathbf{b} \phi_{\mathrm{t}}=0^{\circ}, \phi_{\mathrm{b}}=12^{\circ} ; \mathbf{c} \phi_{\mathrm{t}}=12^{\circ}, \phi_{\mathrm{b}}=12^{\circ}$.

The results related to the time averaged base pressure distribution and wake topology are presented in Fig. 8 and Fig. 9. It is shown that a good horizontal symmetry in the distribution of the mean values of $C_{p}$ is obtained only when the $12^{\circ}$ taper is applied to just the top edge of the base (Fig. 8a). In this case a good balance is reached between the upper and lower pairs of vortices, as the strength of the upper recirculating structures is reduced by the action of the pair of counter rotating trailing vortices forming at the edges of the slanted surface (Fig. 9a) and their cores are moved outwards allowing the formation of a "high" pressure region around the centre of the base. A better pressure recovery is therefore achieved, as highlighted 
by the decrease of 8 counts in the values of $C_{D_{R e a r}}$ ( -12 counts for the rotating case) over the square-back configuration with stationary wheels (although $C_{D_{\text {Rear }}}$ is still 5 counts higher than in the case without wheels). The trailing vortices seem to further contribute to the stabilisation of wake, as suggested by the higher degree of lateral symmetry shown by the time averaged velocity field as well as the shrinking of the regions showing the highest values of the longitudinal and lateral components of the Reynolds stress (Fig. 10). Nevertheless, high values of Tuu and $T_{w}{ }^{*}$ can still be seen corresponding to the inner region of the wake, matching the peak in the values of $R M S\left(\Delta C_{p}\right)$ visible in Fig. 11a. This suggests that a certain degree of "motion" of the reverse flow impinging on the base is still present, although it is more limited than in the previous cases due to the constraints imposed by the combined action of the vortical structures generated by the wheels and the top slant.

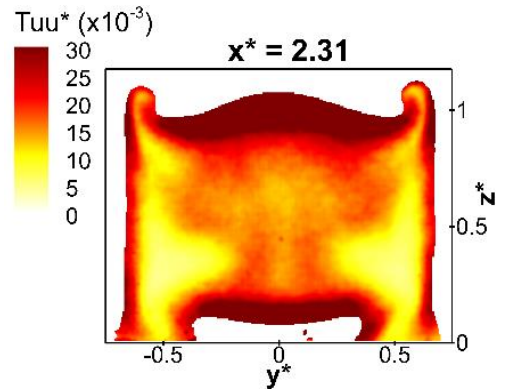

(a)

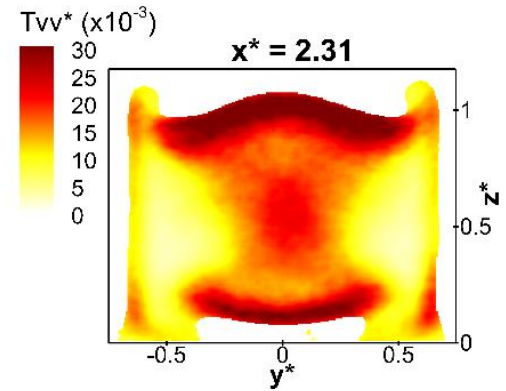

(b)

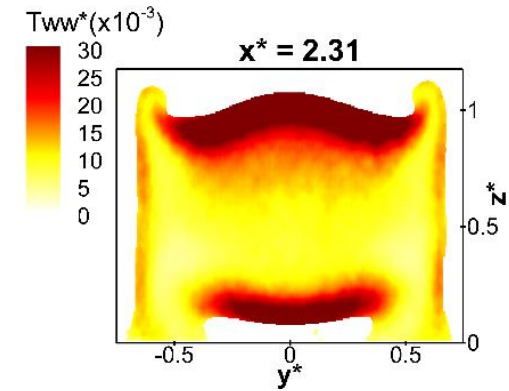

(c)

Fig. 10: Normal components of the Reynolds stress tensor for the configuration with $\phi_{\mathrm{t}}=12^{\circ}, \phi_{\mathrm{b}}=0^{\circ}$ and stationary wheels: $\mathbf{a} \mathrm{T}_{\mathrm{uu}}{ }^{*}=\left\langle\mathrm{u}^{* 2}\right\rangle ; \mathbf{b} \mathrm{T}_{\mathrm{vv}}{ }^{*}=\left\langle\mathrm{v}^{* \prime 2}\right\rangle ; \mathbf{c} \mathrm{T}_{\mathrm{ww}}{ }^{*}=\left\langle\mathrm{w}^{* \prime 2}\right\rangle$.

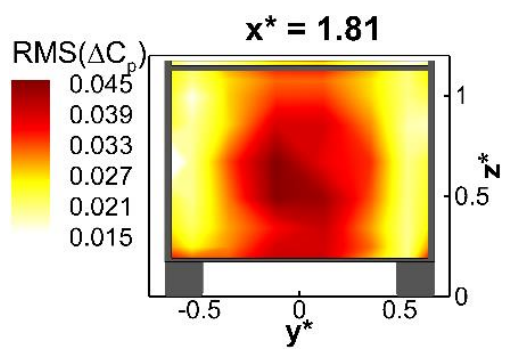

(a)

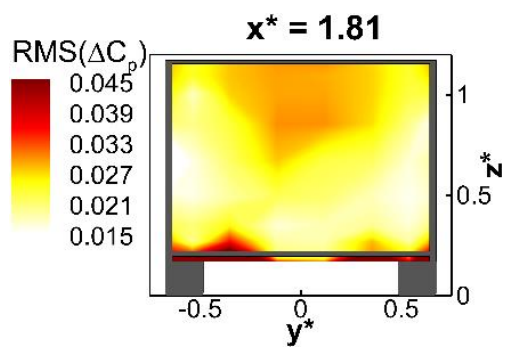

(b)

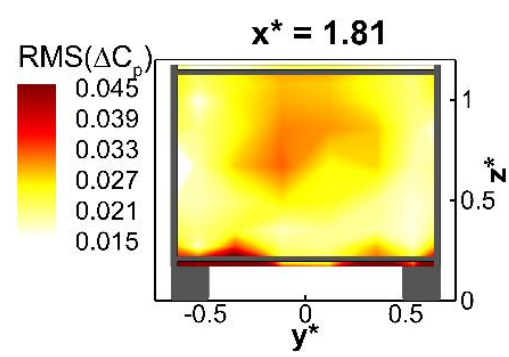

(c)

Fig. 11: Base pressure fluctuations for different rear-end configurations with stationary wheels: a $\phi_{\mathrm{t}}=12^{\circ}, \phi_{\mathrm{b}}=0^{\circ} ; \mathbf{b} \phi_{\mathrm{t}}=0^{\circ}, \phi_{\mathrm{b}}=12^{\circ} ; \mathbf{c} \phi_{\mathrm{t}}=12^{\circ}, \phi_{\mathrm{b}}=12^{\circ}$.

An opposite effect in terms of base pressure distribution is obtained when the $12^{\circ}$ taper is applied only to the bottom trailing edge. In this case, the wake is dominated by the upwash generated by the combined action of the wheels and the diffuser (Fig. 9b). As a result, just a pair of counter rotating vortices can be seen in the wake, extending for almost the entire height of the base. This leads to a further growth in the size of the low pressure region previously described for the square-back case with stationary wheels (Fig. 8b), resulting in a 15 count increase in the value of the area weighted pressure drag over the same configuration (+8 counts for the rotating 
case). At the same time, a significant drop can be seen in the fluctuations of the pressure data, suggesting the disappearance of the bi-stable mode (Fig. 11b).

A similar trend can be seen from the contour plot of $\mathrm{RMS}\left(\Delta \mathrm{C}_{\mathrm{p}}\right)$ obtained for the case with $\phi_{\mathrm{t}}=12^{\circ}$ and $\phi_{\mathrm{b}}=12^{\circ}$ (Fig. 11c). However, the action of the bottom diffuser is largely counteracted by the top slant. Therefore, the results obtained for the time averaged velocity field (Fig. 9c) and the related base pressure distribution (Fig. 8c) are somewhere in between those obtained in the previous two cases. This is further confirmed by the values obtained for $C_{D_{\text {Rear }}}$ which are just 1 count higher than those obtained for the configuration with just the top slant in place, for both the stationary and the rotating case.

\subsection{Effects produced by vertical trailing edge tapers on the RSB mode}

When $12^{\circ}$ tapers are applied to the vertical trailing edges of the model, leaving the horizontal edges squared, a strengthening of the RSB mode can be noticed. In this case, the size of the vortex pair acting in the lower portion of the base is reduced (Fig. 12a) because the pressure difference between the lateral surfaces and the underbody is lowered by the suction introduced by the tapers. As a consequence, the size of the low pressure region is reduced (Fig. 13a), leading to a better pressure recovery, as highlighted by the 21 count reduction obtained for $C_{D_{\text {Rear }}}$ compared to the square-back case with stationary wheels (-23 counts for the rotating case). The weakening of these vortices allows for a higher degree of movement of the upper structures, which results in an increase of the wake unsteadiness seen in both the normal components of the Reynolds stress tensor in the near wake (Fig. 14) and the pressure fluctuations recorded on the base (Fig. 13b). As the wake becomes more unsteady, its sensitivity to any imperfection in the experimental setup is amplified (Evrard et al. [7]), explaining the clear asymmetry that can be found in the time averaged results obtained from both the PIV data (Fig. 12a) and the base pressure data (Fig. 13a). In this case the wake seems to switch between a (left) lateral symmetry breaking (RSB) state (Fig. 12b) and a reflectional symmetry preserving (RSP) state (Fig. 12c), in such a way that the averaged results are clearly biased towards one side. 


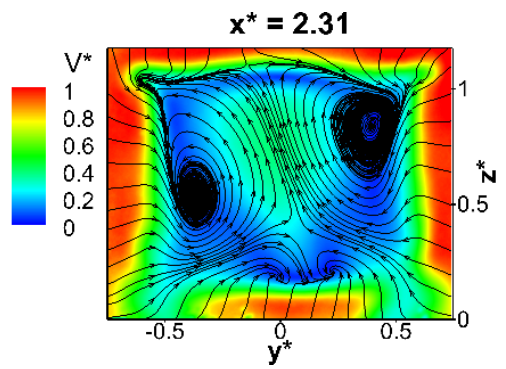

(a)

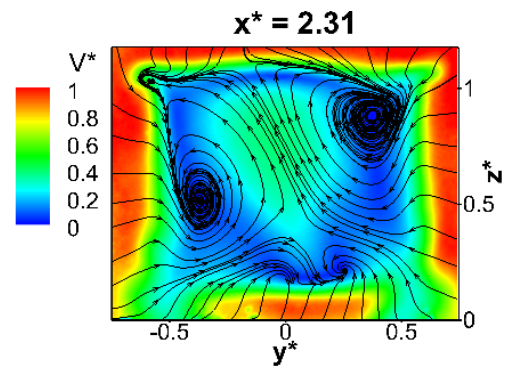

(b)

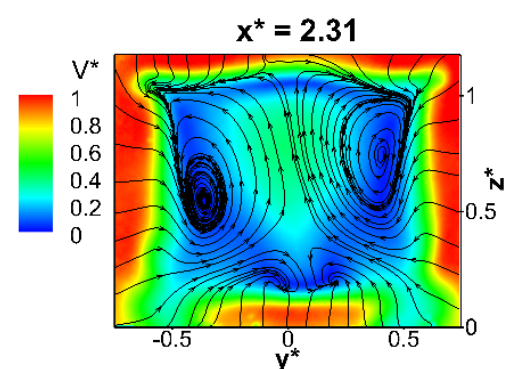

(c)

Fig. 12: Configuration with $\phi_{\mathrm{s}}=12^{\circ}$ and stationary wheels: a time averaged wake topology for $x^{*}=2.31$; $\mathbf{b}$ RSB state and $\mathbf{c}$ RSP state extracted by applying the same method used in Perry et al. [18].

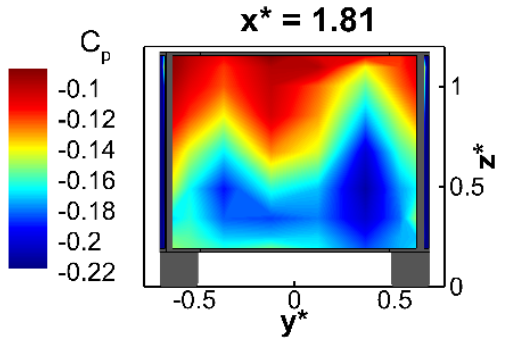

(a)

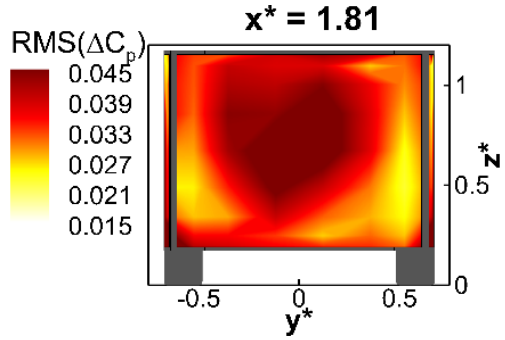

(b)

Fig. 13: Configuration with $\phi_{\mathrm{s}}=12^{\circ}$ and stationary wheels: a time averaged base pressure distribution; $\mathbf{b}$ pressure fluctuations over the model base.

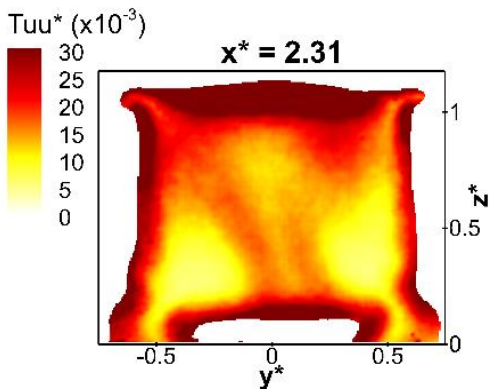

(a)

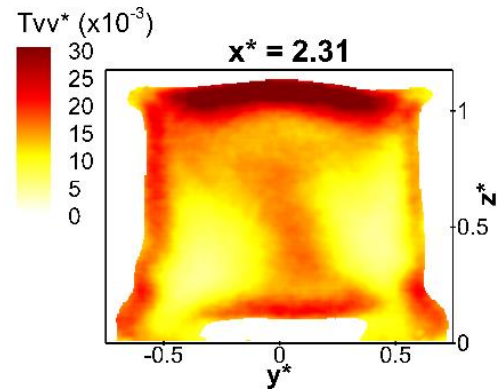

(b)

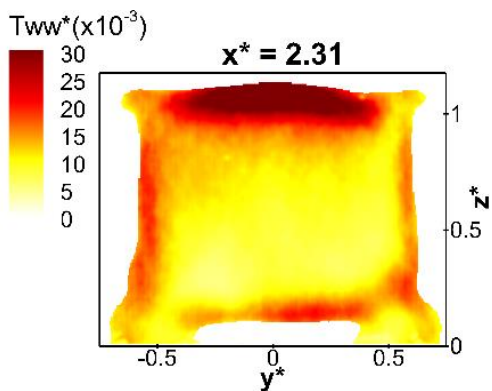

(c)

Fig. 14: Normal components of the Reynolds stress tensor for the model with $\phi_{\mathrm{s}}=12^{\circ}$ and stationary wheels: $\mathbf{a} T_{u u}{ }^{*}=\left\langle u^{* \prime 2}\right\rangle ; \mathbf{b}_{v v}{ }^{*}=\left\langle v^{* \prime 2}\right\rangle ; \mathbf{c} T_{w w}{ }^{*}=\left\langle w^{* \prime 2}\right\rangle$. 


\subsection{Effects of the RSB mode on the side force}

In order to assess the effects of the RSB mode on the lateral component of the aerodynamic force, an approach similar to that used in Perry et al. [18] was followed, considering the probability density function distribution (PDF) distribution of the values recorded for $C_{Y}$ after having resampled the original signal at $5 \mathrm{~Hz}$. The results are presented in Fig. 15.

For the square-back configuration, a clear bi-modal distribution can be seen for the values of $C_{Y}$ when just the front axle is added to the model. No big differences indeed can be seen between this case and the model without wheels tested in Perry et al. [18], confirming the conclusions in §3.1. When all four wheels are added to the geometry, the two peaks seen in the first case disappear, following the weakening of the RSB mode. A high level of dispersion, however, can still be seen for both the stationary and rotating case (Fig. 15a).

The dispersion in the values of $C_{Y}$ is noticeably reduced when the $12^{\circ}$ taper is applied to either the top (Fig. 15b), the bottom (Fig. 15c) or both (Fig. 15d) the horizontal trailing edges. For all these configurations a normal distribution is obtained for the recorded values of the lateral force, in contrast with that seen from the data recorded using the same configurations without wheels. As in other cases no differences appear between the stationary and rotating case.

An opposite trend appears when the tapers are applied to the vertical trailing edges. In this case the level of dispersion associated with the values recorded for $C_{Y}$ increases when adding wheels, further confirming that already stated in $\S 3.3$ for the same configuration.

Similar conclusions can be drawn by looking at the values of the standard deviation $\sigma_{C_{Y}}$ associated to the same signals (Fig. 15f), as suggested by Grandemange et al. [9]. Indeed, the highest values of $\sigma_{C_{Y}}$ are associated with the most bi-stable configurations (namely the square-back without wheels or with just the front axle in place), whilst all the configurations tested with wheels and at least one horizontal taper in place show the lowest level of uncertainty because the effects produced by the RSB mode are much more limited. 


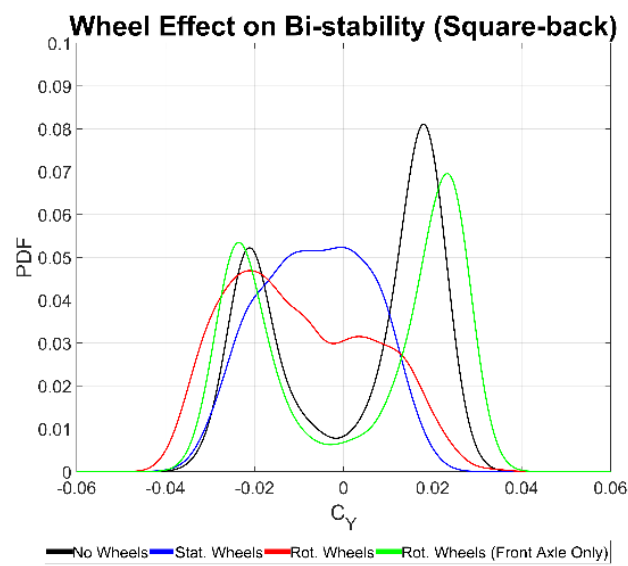

(a)

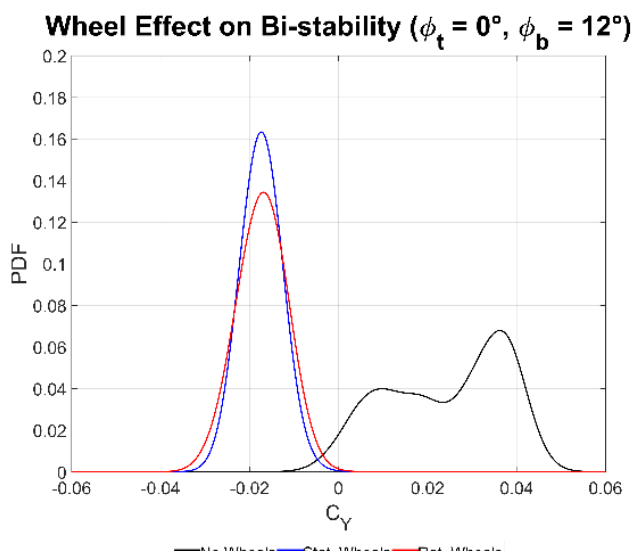

(c)

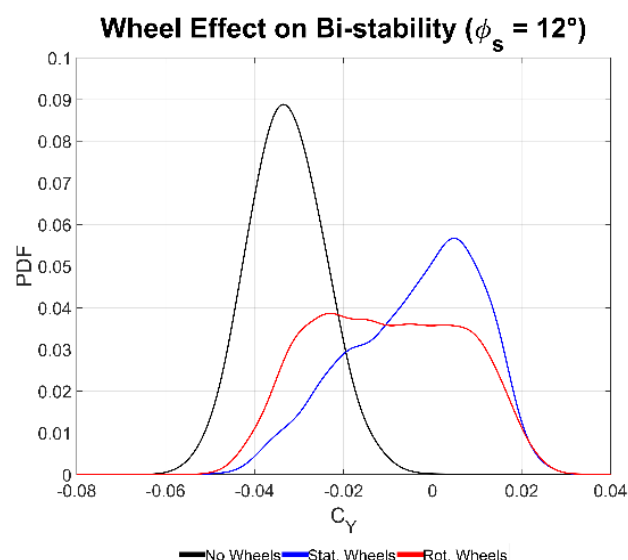

(e)

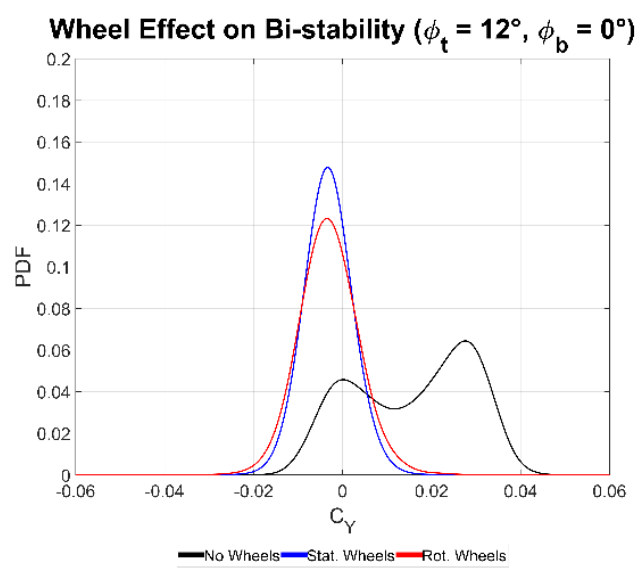

(b)

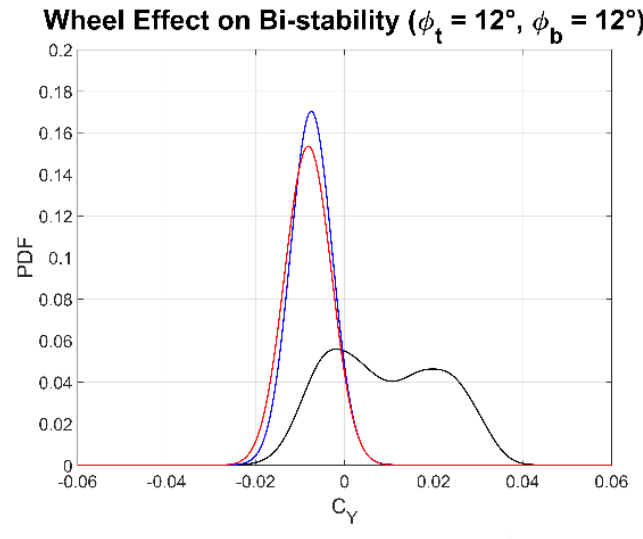

(d)

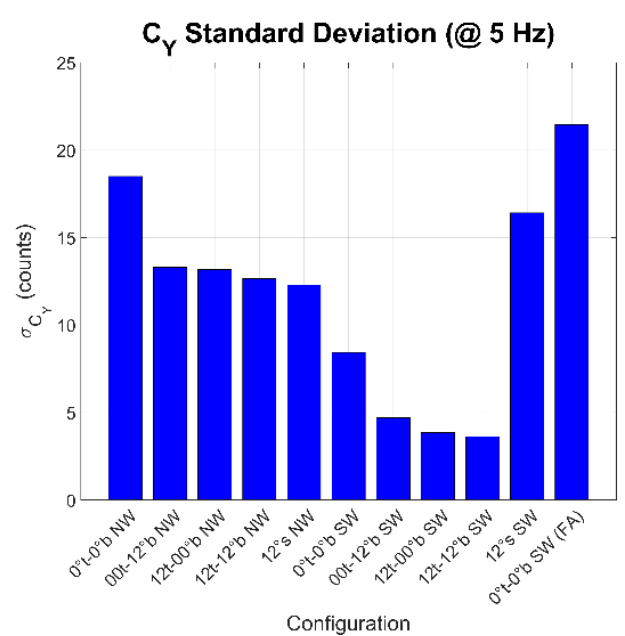

(f)

Fig. 15: PDF distributions of the values of the side force $\mathrm{C}_{\mathrm{Y}}$ resampled at $5 \mathrm{~Hz}$ for different rear-end configurations without wheels and with stationary or rotating wheels: a square-back configuration; $\mathbf{b}$ model with $\phi_{\mathrm{t}}=12^{\circ}$ and $\phi_{\mathrm{b}}=0^{\circ} ; \mathbf{c}$ model with $\phi_{\mathrm{t}}=0^{\circ}$ and $\phi_{\mathrm{b}}=12^{\circ} ; \mathbf{d}$ model with $\phi_{\mathrm{t}}=12^{\circ}$ and $\phi_{\mathrm{b}}=12^{\circ}$; e model with $\phi_{\mathrm{s}}=12^{\circ}$. For each configuration $\mathrm{C}_{\mathrm{Y}}$ has been recorded at the value of the yaw angle $\Psi$ corresponding to the most symmetric base pressure distribution along the lateral direction. $f$ values of the standard deviation of $\mathrm{C}_{\mathrm{Y}}$ recorded for the same configurations either with stationary wheels (SW) 


\section{Summary and conclusions}

In this paper the effects produced by wheels on the base drag and bi-stable RSB mode have been investigated by means of pressure tappings, 2D - 3C PIV and balance measurements. The Windsor body used Perry et al. [18] was considered.

For the square-back configuration, the results showed a general increase in the drag associated with the rear facing surfaces, mainly due to the strengthening of the suction over the base as a consequence of the formation of a pair of counter rotating vortices acting close to the lower portion of the base associated with the gap between the rear wheels. These structures have been shown to counteract the tendency of the upper pair of vortices to alternately align their cores with the model vertical trailing edges, resulting in the bi-stable RSB mode described by Grandemange et al. [11].

The sensitivity of the RSB mode to small changes in the shape of the trailing edges was assessed by applying short high aspect ratio tapers, with a slant angle of $12^{\circ}$, to either the horizontal or the vertical edges of the base. The results showed that, when the slant is applied to the bottom trailing edge, the RSB mode disappears due to the stabilising effect of the upwash generated by the combined action of the wheels and tapered surface. As a consequence, the PDF distribution of the values recorded for the side force switches from a bi-modal distribution to a normal distribution, accompanied with a noticeable reduction of the standard deviation associated with the same data.

A strengthening of the bi-stable mode was seen when the tapers were applied to the side trailing edges, in contrast with the results reported by Pavia et al. [16] for the same model without wheels. At the same time, an improvement in the pressure recovery over the base was noticed.

In all the cases, no significant differences were seen in either the wake topology or the wake dynamics between having the wheels stationary or rotating, apart from a decrease in the values of the drag generated by the rear facing surfaces in the latter case.

Acknowledgements. The authors would like to thank Jaguar Land Rover for the financial support. Thanks are also due to Mr. David Cooper and Mr. Nigel Lines for their excellent work in manufacturing the models and keeping the test facility always in optimal conditions. 


\section{References}

[1] Ahmed, S., Ramm, G., and Faitin, G. (1984). Some salient features of the time-averaged ground vehicle wake. Technical report, Society of Automotive Engineers, Inc., Warrendale, PA.

[2] Barros, D. (2015). Wake and drag manipulation of a bluff body using fluidic forcing. PhD thesis, Ph. D. thesis, École Nationale Supérieure de Mécanique et de Aérotechnique (ENSMA).

[3] Benedict, L. and Gould, R. (1996). Towards better uncertainty estimates for turbulence statistics. Experiments in fluids, 22(2):129-136.

[4] Bonnavion, G., Cadot, O., Évrard, A., Herbert, V., Parpais, S., Vigneron, R., and Délery, J. (2017). On multistabilities of real car's wake. Journal of Wind Engineering and Industrial Aerodynamics, 164:22-33.

[5] Brackston, R., de la Cruz, J. G., Wynn, A., Rigas, G., and Morrison, J. (2016). Stochastic modelling and feedback control of bistability in a turbulent bluff body wake. Journal of Fluid Mechanics, 802:726-749.

[6] Duell, E. G. and George, A. (1999). Experimental study of a ground vehicle body unsteady near wake. Technical report, SAE Technical Paper.

[7] Evrard, A., Cadot, O., Herbert, V., Ricot, D., Vigneron, R., and Délery, J. (2016). Fluid force and symmetry breaking modes of a $3 d$ bluff body with a base cavity. Journal of Fluids and Structures, 61:99-114.

[8] Grandemange, M. (2013). Analysis and control of three-dimensional turbulent wakes: from axisymmetric bodies to road vehicles. PhD thesis, Palaiseau, Ecole polytechnique.

[9] Grandemange, M., Cadot, O., Courbois, A., Herbert, V., Ricot, D., Ruiz, T., and Vigneron, R. (2015). A study of wake effects on the drag of Ahmed's squareback model at the industrial scale. Journal of Wind Engineering and Industrial Aerodynamics, 145:282-291.

[10] Grandemange, M., Gohlke, M., and Cadot, O. (2013a). Bi-stability in the turbulent wake past parallelepiped bodies with various aspect ratios and wall effects. Physics of Fluids (1994-present), 25(9):95-103.

[11] Grandemange, M., Gohlke, M., and Cadot, O. (2013b). Turbulent wake past a three-dimensional blunt body. Part 1. Global modes and bi-stability. Journal of Fluid Mechanics, 722:51-84.

[12] Grandemange, M., Gohlke, M., and Cadot, O. (2014). Turbulent wake past a three-dimensional blunt body. Part 2. Experimental sensitivity analysis. Journal of Fluid Mechanics, 752:439-461.

[13] Johl, G. (2010). The design and performance of a $1.9 \mathrm{mx} 1.3 \mathrm{~m}$ indraft wind tunnel. PhD thesis, @ Guru Johl.

[14] Krajnovic, S. and Davidson, L. (2003). Numerical study of the flow around a bus-shaped body. Journal of Fluids Engineering, 125(3):500-509.

[15] Makihara, T., Kitamura, T., Yamashita, T., Maeda, K., Kato, C., Takayama, T., Yamamoto, K., Yamade, Y., and Suzuki, Y. (2016). Identification of vortical structure that drastically worsens aerodynamic drag on a 2-box vehicle using 
large-scale simulations. SAE International Journal of Passenger CarsMechanical Systems, 9(2016-01-1585).

[16] Pavia, G., Passmore, M., and Gaylard, A. (2016). Influence of short rear end tapers on the unsteady base pressure of a simplified ground vehicle. Technical report, SAE Technical Paper.

[17] Perry, A.-K., Almond, M., Passmore, M., and Littlewood, R. (2016a). The study of a bi-stable wake region of a generic squareback vehicle using tomographic piv. SAE International Journal of Passenger Cars-Mechanical Systems, 9(2016-01-1610).

[18] Perry, A.-K., Pavia, G., and Passmore, M. (2016b). Influence of short rear end tapers on the wake of a simplified square-back vehicle: wake topology and rear drag. Experiments in Fluids, 57(11):169

[19] Prasad, A. K. (2000). Stereoscopic particle image velocimetry. Experiments in fluids, 29(2):103-116.

[20] SAE (2010). Surface vehicle recommended practice. Technical Report J1594, SAE International.

[21] Sims-Williams, D. B. and Dominy, R. (1998). Experimental investigation into unsteadiness and instability in passenger car aerodynamics. Technical report, SAE Technical Paper

[22] Varon, E., Eulalie, Y., Edwige, S., Gilotte, P., and Aider, J.-L. (2017). Chaotic dynamics of large-scale structures in a turbulent wake. Physical Review Fluids, 2(3):034604.

[23] Volpe, R., Devinant, P., and Kourta, A. (2015). Experimental characterization of the unsteady natural wake of the full-scale square back Ahmed body: flow bi-stability and spectral analysis. Experiments in Fluids, 56(5):1-22.

[24] Willert, C. E. and Gharib, M. (1991). Digital particle image velocimetry. Experiments in fluids, 10(4):181-193.

[25] Wood, D. (2015). The effect of rear geometry changes on the notchback flow field. PhD thesis, ๑ Daniel Wood. 